\title{
Profinite Monads, Profinite Equations, and Reiterman's Theorem
}

\author{
Liang-Ting Chen ${ }^{1, \star}$, Jiř́ Adámek ${ }^{1}$, Stefan Milius ${ }^{2, \star \star}$, and Henning Urbat ${ }^{1, \star \star \star}$ \\ 1 Institut für Theoretische Informatik \\ Technische Universität Braunschweig, Germany \\ 2 Lehrstuhl für Theoretische Informatik \\ Friedrich-Alexander Universität Erlangen-Nürnberg, Germany
}

\begin{abstract}
Profinite equations are an indispensable tool for the algebraic classification of formal languages. Reiterman's theorem states that they precisely specify pseudovarieties, i.e. classes of finite algebras closed under finite products, subalgebras and quotients. In this paper Reiterman's theorem is generalised to finite Eilenberg-Moore algebras for a monad $\mathbf{T}$ on a variety $\mathscr{D}$ of (ordered) algebras: a class of finite $\mathbf{T}$-algebras is a pseudovariety iff it is presentable by profinite (in-)equations. As an application, quasivarieties of finite algebras are shown to be presentable by profinite implications. Other examples include finite ordered algebras, finite categories, finite $\infty$-monoids, etc.
\end{abstract}

\section{Introduction}

Algebraic automata theory investigates the relationship between the behaviour of finite machines and descriptions of these behaviours in terms of finite algebraic structures. For example, regular languages of finite words are precisely the languages recognised by finite monoids. And Schützenberger's theorem [28] shows that star-free regular languages correspond to aperiodic finite monoids, which easily leads to the decidability of star-freeness. A generic correspondence result of this kind is Eilenberg's variety theorem 13. It gives a bijective correspondence between varieties of languages (classes of regular languages closed under boolean operations, derivatives and homomorphic preimages) and pseudovarieties of monoids (classes of finite monoids closed under finite products, submonoids and quotients). Another, more syntactic, characterisation of pseudovarieties follows from Reiterman's theorem [25] (see also Banaschewski 8]): they are precisely the classes of finite monoids specified by profinite equations.

In the meantime Eilenberg-type correspondences have been discovered for other kinds of algebraic structures, including ordered monoids [21], idempotent

${ }^{\star}$ Liang-Ting Chen acknowledges gratefully partial support from AFOSR.

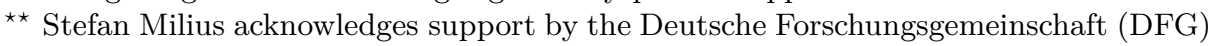
under project MI $717 / 5-1$

*** Jiří Adámek and Henning Urbat acknowledge support by the Deutsche Forschungsgemeinschaft (DFG) under project AD 187/2-1 
semirings [23, associative algebras over a field [26] and Wilke algebras [30, always with rather similar proofs. This has spurred recent interest in generic approaches to algebraic language theory that can produce such correspondences as instances of a single result. Bojańczyk [1] extends the classical notion of language recognition by monoids (viewed as algebraic structures over the category of sets) to algebras for an arbitrary monad on many-sorted sets. He also presents an Eilenberg-type theorem at this level of generality, interpreting a result of Almeida 7 in categorical terms. Our previous work in 2 2, 4, 12, takes an orthogonal approach: one keeps monoids but considers them in categories $\mathscr{D}$ of (ordered) algebras such as posets, semilattices and vector spaces. Analysing the latter work it becomes clear that the step from sets to more general categories $\mathscr{D}$ is necessary to obtain the right notion of language recognition by finite monoids; e.g. to cover Polák's Eilenberg-type theorem for idempotent semirings [23], one needs to take the base category $\mathscr{D}$ of semilattices. On the other hand, from Bojańczyk's work it is clear that one also has to generalise from monoids to other algebraic structures if one wants to capture such examples as Wilke algebras.

The present paper is the first step in a line of work that considers a common roof for both approaches, working with algebras for a monad $\mathbf{T}$ on an arbitrary variety $\mathscr{D}$ of many-sorted, possibly ordered algebras.

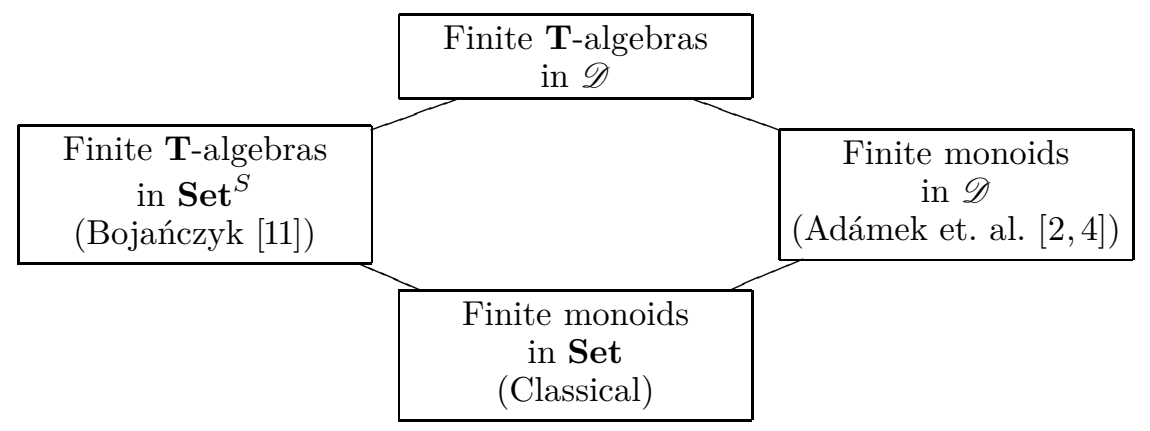

Our main contribution is a generalisation of Reiterman's theorem, stating that pseudovarieties of finite algebras are presentable by profinite equations, to the more general situation of algebras for a monad. Starting with a variety $\mathscr{D}$, we form the pro-completion of the full subcategory $\mathscr{D}_{f}$ of finite algebras,

$$
\widehat{\mathscr{D}}:=\text { Pro- } \mathscr{D}_{f} \text {. }
$$

For example, for $\mathscr{D}=$ sets, posets and monoids we get $\widehat{\mathscr{D}}=$ Stone spaces, Priestley spaces and profinite monoids. Next, we consider a monad $\mathbf{T}$ on $\mathscr{D}$ and associate to it a monad $\widehat{\mathbf{T}}$ on $\widehat{\mathscr{D}}$, called the profinite monad of $\mathbf{T}$. For example, if $\mathscr{D}=$ Set and $\mathbf{T}$ is the finite word monad (whose algebras are precisely monoids), then $\widehat{\mathbf{T}}$ is the monad of profinite monoids on the category of Stone spaces; that is, $\widehat{\mathbf{T}}$ associates to each finite Stone space (= finite set) $X$ the space $\widehat{X^{*}}$ of profinite words on $X$. Similarly, for the monad $\mathbf{T}$ of finite and infinite words on Set (whose algebras we call $\infty$-monoids) the profinite monad $\widehat{\mathbf{T}}$ constructs the space of profinite $\infty$-words. 
The classical profinite equations for monoids, used for presenting pseudovarieties of monoids, are generalised to profinite equations $u=v$ that are pairs of elements of $\widehat{\mathbf{T}} \hat{\Phi_{X}}$, where $\hat{\Phi_{X}}$ is the free profinite $\mathscr{D}$-algebra on a finite set $X$ of variables. Our main result is that profinite equations present precisely classes of finite T-algebras closed under finite products, subalgebras, and quotients.

We will additionally study a somewhat unusual concept of profinite equation where in lieu of finite sets $X$ of variables we use finite algebras $X \in \mathscr{D}_{f}$ of variables. The classes of finite $\mathbf{T}$-algebras presented by such profinite equations are then precisely those closed under finite products, subalgebras, and split quotients. These two variants are actually instances of a general result (Theorem 4.12) that is parametric in a class $\mathscr{X}$ of "algebras of variables" in $\mathscr{D}$.

The above results hold if $\mathscr{D}$ is a variety of algebras. In case that $\mathscr{D}$ is a variety of ordered algebras, we obtain the analogous two results, working with profinite inequations $u \leq v$ instead of equations. As instances we recover Reiterman's original theorem [25] and its version for ordered algebras due to Pin and Weil [22]. Another consequence of our theorem is the observation that quasivarietes of finite algebras in $\mathscr{D}$, i.e. subclasses of $\mathscr{D}_{f}$ closed under finite products and subalgebras, are presentable by profinite implications. Moreover, we obtain a number of new Reiterman-type results. For example, for the monad of finite and infinite words on Set, our Reiterman theorem shows that a class of finite $\infty$-monoids is a pseudovariety iff it can be presented by equations between profinite $\infty$-words. Finally, we can also treat categories of $\mathbf{T}$-algebras that are not varieties. E.g. by taking for $\mathscr{D}$ the category of graphs and $\mathbf{T}$ the free-category monad we essentially recover a result of Jones on pseudovarieties of finite categories [17].

\section{Preliminaries}

In this section we review the necessary concepts from category theory, universal algebra and topology we will use throughout the paper. Recall that for a finitary many-sorted signature $\Gamma$ a variety of $\Gamma$-algebras is a full subcategory of $\mathrm{Alg}_{\Gamma}$, the category of $\Gamma$-algebras, specified by equations $s=t$ between $\Gamma$-terms. By Birkhoff's HSP theorem varieties are precisely the classes of algebras closed under products, subalgebras, and quotients (= homomorphic images). Similarly, ordered $\Gamma$-algebras are posets equipped with order-preserving $\Gamma$-operations, and their morphisms are order-preserving $\Gamma$-homomorphisms. A quotient of an ordered algebra $B$ is represented by a surjective morphism $e: B \rightarrow A$, and a subalgebra of $B$ is represented by an order-reflecting morphism $m: A \longmapsto B$, i.e. $m x \leq m y$ iff $x \leq y$. A variety of ordered $\Gamma$-algebras is a full subcategory of $\mathrm{Alg}_{\leq \Gamma}$, the category of ordered $\Gamma$-algebras, specified by inequations $s \leq t$ between $\Gamma$ terms. By Bloom's HSP theorem [10, varieties of ordered algebras are precisely the classes of ordered algebras closed under products, subalgebras and quotients.

Remark 2.1. For notational simplicity we restrict our attention to single-sorted varieties. However, all definitions, theorems and proofs that follow are easily adapted to a many-sorted setting. See also Remark 5.7 and Example 5.8. 
Definition 2.2. Let $\mathscr{D}$ be a variety of algebras or ordered algebras.

(a) A topological $\mathscr{D}$-algebra is a topological space endowed with a $\mathscr{D}$-algebraic structure such that every operation is continuous with respect to the product topology. Morphisms of topological $\mathscr{D}$-algebras are continuous $\mathscr{D}$-morphisms.

(b) A topological $\mathscr{D}$-algebra is profinite if it is a cofiltered limit of finite $\mathscr{D}$ algebras with discrete topology.

Notation 2.3. Throughout this paper we fix a variety $\mathscr{D}$ of algebras or ordered algebras, equipped with the factorisation system of quotients and subalgebras. We denote by $\mathscr{D}$ the category of profinite $\mathscr{D}$-algebras. We use the forgetful functors

$$
\mathscr{D}_{f} \stackrel{\hat{J}}{\longrightarrow} \widehat{\mathscr{D}} \stackrel{V}{\longrightarrow} \mathscr{D}
$$

where $V$ forgets the topology and $\hat{J}$ views a finite $\mathscr{D}$-algebra as a profinite $\mathscr{D}$ algebra with discrete topology. We will often identify $A \in \mathscr{D}_{f}$ with $\hat{J} A$.

Example 2.4. 1. $\widehat{\text { Set }}$ is the category Stone of Stone spaces, i.e. compact spaces such that any two distinct elements can be separated by a clopen set.

2. Let Pos be the category of posets and monotone maps, viewed as the variety of ordered algebras over the empty signature. Then $\widehat{\text { Pos }}$ is the category Priest of Priestley spaces 24, i.e. ordered compact spaces such that for any two elements $u, v$ with $u \not \leq v$ there is a clopen upper set containing $u$ but not $v$.

3. For the variety Mon of monoids, the category $\widehat{\text { Mon }}$ consists of all monoids in Stone; that is, a topological monoid is profinite iff it carries a Stone topology. Analogous descriptions of $\widehat{D}$ hold for most familiar varieties $\mathscr{D}$ over a finite signature, e.g. groups, semilattices, vector spaces over a finite field; see [16].

Remark 2.5. By [16, Remark VI.2.4] the category $\widehat{\mathscr{D}}$ is the pro-completion, i.e. the free completion under cofiltered limits, of $\mathscr{D}_{f}$. Hence $\widehat{\mathscr{D}}$ is dual to a locally finitely presentable category [6], which entails the following properties:

(i) Every object $A$ of $\widehat{\mathscr{D}}$ is the cofiltered limit of all morphisms $h: A \rightarrow A^{\prime}$ with finite codomain. More precisely, if $\left(A \downarrow \mathscr{D}_{f}\right)$ denotes the comma category of all such morphisms $h$, the diagram

$$
\left(A \downarrow \mathscr{D}_{f}\right) \rightarrow \widehat{\mathscr{D}}, \quad h \mapsto A^{\prime},
$$

has the limit $A$ with limit projections $h$.

(ii) Given a cofiltered limit cone $\pi_{i}: A \rightarrow A_{i}(i \in I)$ in $\widehat{\mathscr{D}}$, any morphism $f: A \rightarrow B$ with finite codomain factors through some $\pi_{i}$.

Lemma 2.6. $\widehat{\mathscr{D}}$ has the factorisation system of surjective morphisms and injective (resp. order-reflecting) morphisms. 
Definition 2.7. The profinite completion of an object $D \in \mathscr{D}$ is the limit $\hat{D} \in \widehat{\mathscr{D}}$ of the cofiltered diagram

$$
\left(D \downarrow \mathscr{D}_{f}\right) \rightarrow \widehat{\mathscr{D}}, \quad\left(h: D \rightarrow D^{\prime}\right) \mapsto D^{\prime}
$$

We denote the limit projection corresponding to $h: D \rightarrow D^{\prime}$ by $\hat{h}: \hat{D} \rightarrow D^{\prime}$. Observe that $\hat{D}=D$ for any $D \in \mathscr{D}_{f}$, and $\widehat{h}=h$ for any morphism $h$ in $\mathscr{D}_{f}$.

Proposition 2.8. The maps $D \mapsto \hat{D}$ and $h \mapsto \hat{h}$ extend to a left adjoint for the forgetful functor $V$, denoted by

$$
\hat{\imath}: \mathscr{D} \rightarrow \widehat{\mathscr{D}} \text {. }
$$

Remark 2.9. We will frequently use the following facts:

(a) Homomorphism theorem. Given morphisms $e: A \rightarrow B$ and $f: A \rightarrow C$ in $\mathscr{D}$ with $e$ surjective, there exists a morphism $f^{\prime}$ with $f^{\prime} \cdot e=f$ iff $e(a)=e\left(a^{\prime}\right)$ implies $f(a)=f\left(a^{\prime}\right)$ (resp. $e(a) \leq e\left(a^{\prime}\right)$ implies $\left.f(a) \leq f\left(a^{\prime}\right)\right)$ for all $a, a^{\prime} \in A$. Moreover, if $A, B, C$ are topological $\mathscr{D}$-algebras with a compact Hausdorff topology and $e$ and $f$ are continuous $\mathscr{D}$-morphisms, then $f^{\prime}$ is continuous.

(b) The forgetful functor $|-|: \mathscr{D} \rightarrow$ Set has a left adjoint assigning to each set $X$ the free $\mathscr{D}$-algebra $\Phi_{X}$ on $X$.

(c) Free $\mathscr{D}$-algebras are projective: for any morphism $f: \Phi_{X} \rightarrow B$ and any surjective morphism $e: A \rightarrow B$ in $\mathscr{D}$ there exists a morphism $f^{\prime}: \Phi_{X} \rightarrow A$ with $e \cdot f^{\prime}=f$. Indeed, choose a function $m:|B| \rightarrow|A|$ with $e \cdot m=i d$. Then the restriction of $m \cdot f$ to $X$ extends to a morphism $f^{\prime}: \Phi_{X} \rightarrow A$ of $\mathscr{D}$ with $f=f^{\prime} \cdot e$, since the morphisms on both sides agree on the generators $X$.

Notation 2.10. For a monad $\mathbf{T}=(T, \eta, \mu)$ on $\mathscr{D}$, we write $\mathscr{D}^{\mathbf{T}}$ for the category of $\mathbf{T}$-algebras and $\mathbf{T}$-homomorphisms, and $\mathscr{D}_{f}^{\mathbf{T}}$ for the full subcategory of finite T-algebras. The forgetful functors are denoted by

$$
U: \mathscr{D}_{f}^{\mathbf{T}} \rightarrow \mathscr{D}_{f} \quad \text { and } \quad U^{\mathbf{T}}: \mathscr{D}^{\mathbf{T}} \rightarrow \mathscr{D} .
$$

Recall that $U^{\mathbf{T}}$ has a left adjoint mapping $D \in \mathscr{D}$ to its free $\mathbf{T}$-algebra $\left(T D, \mu_{D}\right)$.

Remark 2.11. If $T$ preserves surjective morphisms, the homomorphism theorem applies to $\mathbf{T}$-algebras. That is, if $A, B, C$ in Remark 2.9)(a) are $\mathbf{T}$-algebras and $e$ and $f$ are $\mathbf{T}$-homomorphisms, so is $f^{\prime}$. Moreover the factorisation system of $\mathscr{D}$ lifts to $\mathscr{D}^{\mathbf{T}}$ : every $\mathbf{T}$-homomorphism $h:(A, \alpha) \rightarrow(B, \beta)$ can be factorised into a surjective $\mathbf{T}$-homomorphism followed by an injective (resp. order-reflecting) one. Quotients and subalgebras of $\mathbf{T}$-algebras are taken w.r.t. this factorisation system.

Example 2.12. We are mainly interested in monads representing structures in algebraic language theory. 
(a) Finite words. The classical example is the free-monoid monad $\mathbf{T}$ on $\mathscr{D}=\mathbf{S e t}$,

$$
T X=X^{*}=\coprod_{n<\omega} X^{n} .
$$

The importance of the monad $\mathbf{T}$ is that functions $T X \rightarrow\{0,1\}$ correspond to languages of finite words over the alphabet $X$, and regular languages are precisely the languages recognized by finite $\mathbf{T}$-algebras (= finite monoids). Bojańczyk [1] recently gave a generalisation of the classical Eilenberg theorem to arbitrary monads $\mathbf{T}$ on Set, relating pseudovarieties of finite $\mathbf{T}$-algebras to varieties of $\mathbf{T}$-recognisable languages.

(b) Finite words over semilattices. From the perspective of algebraic language theory it is natural to study monoids in algebraic categories beyond Set. For example, let $\mathscr{D}=\mathbf{J S L}$ be the variety of join-semilattices with 0 , considered as a monoidal category w.r.t. the usual tensor product. The free-monoid monad on JSL is given by

$$
T X=X^{\circledast}=\coprod_{n<\omega} X^{\otimes n},
$$

the coproduct of all finite tensor powers of $X$, and $\mathbf{T}$-algebras are precisely idempotent semirings. In case $X=\mathcal{P}_{f} X_{0}$ is the free semilattice on a set $X_{0}$ one has $T X=\mathcal{P}_{f} X_{0}^{*}$, the semilattice of all finite languages over $X_{0}$. Hence semilattice morphisms from $T X$ into the two-chain $0<1$ correspond again to formal languages over $X_{0}$. This setting allows one to study disjunctive varieties of languages in the sense of Polák [23, see 22 4]. Note that although the variety of idempotent semirings can also be represented by the free idempotent semiring monad $T^{\prime} X=\mathcal{P}_{f} X^{*}$ on Set, functions from $T^{\prime} X=\mathcal{P}_{f} X^{*}$ to $\{0,1\}$ do not correpond to formal languages over $X$.

(c) Infinite words. The monad

$$
T X=X^{\infty}=X^{*}+X^{\omega}
$$

on $\mathscr{D}=$ Set represents languages of finite and infinite words. The unit $\eta_{X}: X \rightarrow X^{*}$ is given by inclusion, and the multiplication $\mu_{X}:\left(X^{\infty}\right)^{\infty} \rightarrow$ $X^{\infty}$ is concatentation: $\mu_{X}\left(w_{0} w_{1} w_{2} \ldots\right)=w_{0} w_{1} w_{2} \ldots$ if all words $w_{i}$ are finite, and otherwise $\mu_{X}\left(w_{0} w_{1} w_{2} \ldots\right)=w_{0} w_{1} w_{2} \ldots w_{j}$ for the smallest $j$ with $w_{j}$ infinite. T-algebras are $\infty$-monoids, i.e. monoids with an additional $\omega$-ary multiplication and the expected mixed associative laws. Again, functions from $T X$ to $\{0,1\}$ correspond to languages (of finite and infinite words), and $\omega$-regular languages are precisely the languages recognised by finite $\infty$-monoids. This was observed by Bojańczyk [11, who also derived an Eilenberg-type theorem for varieties of $\omega$-regular languages and pseudovarieties of $\infty$-monoids along the lines of Wilke [30. As in (b) one can replace $\infty$-monoids in Set by "idempotent $\infty$-semirings", viewed as algebras for a suitable monad on JSL, and thus extend Polák's theorem [23] from finite word languages to $\omega$-regular languages. We leave the details for future work. 
(d) In contrast to the previous examples, the category $\mathscr{D}^{\mathbf{T}}$ is not always monadic over Set resp. Pos. To see this, let $\mathscr{D}=$ Set $_{0,1}$ be the variety of sets with two constants, that is, the category of all algebras over the signature with two constant symbols 0,1 . The full subcategory $\mathbf{S e t}_{0 \neq 1}$, consisting of singletons and sets with distinct constants $0 \neq 1$, is reflective and hence monadic over Set $_{0,1}$. However, it is not monadic over Set.

\section{Profinite Monads}

In this section we introduce profinite monads, our main tool for the investigation of profinite equations and Reiterman's theorem for T-algebras in Section 4 .

Assumption 3.1. As in the previous section let $\mathscr{D}$ be a variety of algebras or ordered algebras. Moreover, let $\mathbf{T}=(T, \eta, \mu)$ be a monad on $\mathscr{D}$ such that $T$ preserves surjective morphisms.

Recall that the right Kan extension of a functor $F: \mathscr{A} \rightarrow \mathscr{C}$ along $K: \mathscr{A} \rightarrow \mathscr{B}$ is a functor $R: \mathscr{B} \rightarrow \mathscr{C}$ with a universal natural transformation $\varepsilon: R K \rightarrow F$, i.e. for every functor $G: \mathscr{B} \rightarrow \mathscr{C}$ and every natural transformation $\gamma: G K \rightarrow F$ there exists a unique natural transformation $\gamma^{\dagger}: G \rightarrow R$ with $\gamma=\varepsilon \cdot \gamma^{\dagger} K$. In case $F=K$, the functor $R$ carries a natural monad structure: the unit is given by $\hat{\eta}=\left(i d_{K}\right)^{\dagger}:$ Id $\rightarrow R$ and the multiplication by $\hat{\mu}=(\varepsilon \cdot R \varepsilon)^{\dagger}: R R \rightarrow R$. The monad $(R, \hat{\eta}, \hat{\mu})$ is called the codensity monad of $K$, see e.g., [19].

Definition 3.2. The profinite monad of $\mathbf{T}$ is the codensity $\operatorname{monad} \widehat{\mathbf{T}}=(\hat{T}, \hat{\eta}, \hat{\mu})$ of the functor

$$
K=\hat{J} U: \mathscr{D}_{f}^{\mathbf{T}} \rightarrow \mathscr{D}_{f} \rightarrow \widehat{\mathscr{D}} .
$$

Remark 3.3. A related concept was recently studied by Bojańczyk 11] who associates to every monad $\mathbf{T}$ on Set a monad $\overline{\mathbf{T}}$ on Set (rather than $\widehat{\mathbf{S e t}}=$ Stone as in our setting!). Specifically, $\overline{\mathbf{T}}$ is the monad induced by the composite right adjoint Stone ${ }^{\widehat{T}} \rightarrow$ Stone $\stackrel{V}{\rightarrow}$ Set. Its construction also appears in the work of Kennison and Gildenhuys [18] who investigated codensity monads for Set-valued functors and their connection with profinite algebras.

Remark 3.4. (a) One can compute $\hat{T} X$ for $X \in \widehat{\mathscr{D}}$ via the limit formula for right Kan extensions, see e.g. [20, Theorem X.3.1]. Letting $(X \downarrow \hat{J} U)$ denote the comma category of all arrows $f: X \rightarrow A$ with $(A, \alpha) \in \mathscr{D}_{f}^{\mathbf{T}}$, the object $\hat{T} X$ is the limit of the diagram

$$
(X \downarrow \hat{J} U) \rightarrow \widehat{\mathscr{D}}, \quad f \mapsto A .
$$

(b) For $D \in \mathscr{D}$ a morphism $f: \hat{D} \rightarrow A$ with $(A, \alpha) \in \mathscr{D}_{f}^{\mathbf{T}}$ corresponds to a $\mathbf{T}$ homomorphism $h:\left(T D, \mu_{D}\right) \rightarrow(A, \alpha)$, since $\left(T D, \mu_{D}\right)$ is the free $\mathbf{T}$-algebra on $D$. Hence to compute $\hat{T} \hat{D}$ one can replace $(\hat{D} \downarrow \hat{J} U)$ by the category of all $h:\left(T D, \mu_{D}\right) \rightarrow(A, \alpha)$ with $(A, \alpha) \in \mathscr{D}_{f}^{\mathbf{T}}$. We denote the limit cone by

$$
h^{+}: \hat{T} \hat{D} \rightarrow \hat{A} \text {. }
$$

One can restrict the diagram defining $\hat{T} \hat{D}$ to surjective T-homomorphisms: 
Proposition 3.5. For all $D \in \mathscr{D}$ the object $\hat{T} \hat{D}$ is the cofiltered limit of all finite T-algebra quotients e: $\left(T D, \mu_{D}\right) \rightarrow(A, \alpha)$.

Example 3.6 (Profinite words). For the monad $T X=X^{*}$ on $\mathscr{D}=$ Set the profinite monad $\widehat{\mathbf{T}}$ assigns to every finite set (= finite Stone space) $X$ the space $\hat{T} X=\widehat{X^{*}}$ of profinite words over $X$. This is the limit in Stone of all finite (discrete) quotient monoids of $X^{*}$. Similarly, for $T X=X^{\infty}$ the profinite monad $\widehat{\mathbf{T}}$ constructs the space $\hat{T} X$ of "profinite $\infty$-words" over $X$.

Lemma 3.7. (a) $\hat{T}$ preserves cofiltered limits and surjections.

(b) Given a cofiltered limit cone $h_{i}: A \rightarrow A_{i}(i \in I)$ in $\widehat{\mathscr{D}} \widehat{\mathbf{T}}$, any $\widehat{\mathbf{T}}$-homomorphism $h: A \rightarrow B$ with finite codomain factors through some $h_{i}$.

Remark 3.8. (a) Since $\hat{T}$ preserves surjections, the factorisation system of $\widehat{\mathscr{D}}$ lifts to $\widehat{\mathscr{D}} \widehat{\mathbf{T}}$, so we can speak about quotients and subalgebras of $\widehat{\mathbf{T}}$-algebras. Moreover, the homomorphism theorem holds for $\widehat{\mathbf{T}}$-algebras, cf. Remark 2.11.

(b) Lemma 3.7 (b) exhibits a crucial technical difference between our profinite monad $\widehat{\mathbf{T}}$ and Bojańczyk's $\overline{\mathbf{T}}$, see Remark 3.3 . For example, for the identity monad $\mathbf{T}$ on Set, the monad $\overline{\mathbf{T}}$ is the ultrafilter monad whose algebras are compact Hausdorff spaces, and the factorisation property in the lemma fails.

Remark 3.9. For each finite T-algebra $(A, \alpha)$ the morphism $\alpha$ is itself a $\mathbf{T}$ homomorphism $\alpha:\left(T A, \mu_{A}\right) \rightarrow(A, \alpha)$, and thus yields the limit projection

$$
\alpha^{+}: \hat{T} \hat{A} \rightarrow \hat{A}
$$

of (3.1). The unit $\hat{\eta}_{\hat{D}}$ and multiplication $\hat{\mu}_{\hat{D}}$ of $\widehat{\mathbf{T}}$ are determined by the following commutative diagrams for all T-homomorphisms $h:\left(T D, \mu_{D}\right) \rightarrow(A, \alpha)$ :
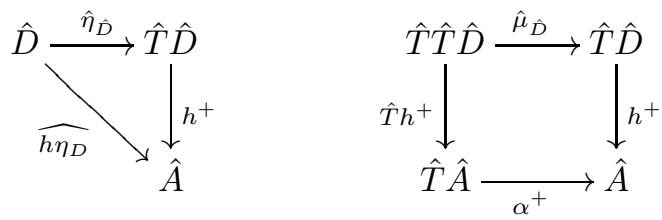

Hence $\left(\hat{A}, \alpha^{+}\right)$is a $\widehat{\mathbf{T}}$-algebra: the unit and associative law for $\widehat{\mathbf{T}}$-algebras follow by putting $D=A$ and $h=\alpha$ in (3.2). Moreover, (3.2) states precisely that $h^{+}:\left(\hat{T} \hat{D}, \hat{\mu}_{\hat{D}}\right) \rightarrow\left(A, \alpha^{+}\right)$is the unique $\widehat{\mathbf{T}}$-homomorphism extending the map $\widehat{h \eta_{D}}$ for every $h$ as above.

Proposition 3.10. The maps $(A, \alpha) \mapsto\left(\hat{A}, \alpha^{+}\right)$and $h \mapsto \widehat{h}$ define an isomorphism between the categories of finite $\mathbf{T}$-algebras and finite $\widehat{\mathbf{T}}$-algebras:

$$
\mathscr{D}_{f}^{\mathbf{T}} \cong \widehat{\mathscr{D}} \widehat{\mathbf{T}} .
$$




\section{Reiterman's Theorem for T-Algebras}

Reiterman's theorem [8, 25] states that, for any variety $\mathscr{D}$ of algebras, a class of finite algebras in $\mathscr{D}$ is a pseudovariety, i.e. closed under finite products, subobjects and quotients, iff it is presented by profinite equations. Later Pin and Weil 22] proved the corresponding result for varieties $\mathscr{D}$ of ordered algebras: pseudovarieties are precisely the classes of finite algebras in $\mathscr{D}$ presented by profinite inequations. In our categorical setting these two theorems represent the case where $\mathbf{T}$ is chosen to be the identity monad on $\mathscr{D}$. In Section 4.1 we introduce pseudovarieties and profinite (in-)equations for arbitrary monads $\mathbf{T}$ on $\mathscr{D}$, a straightforward extension of the original notions. In Section 4.2 we present a further generalisation and prove the main result of this paper, Reiterman's theorem for finite $\mathbf{T}$-algebras.

\subsection{Pseudovarieties and profinite (in-)equations}

Let us start with extending the classical concept of a pseudovariety to $\mathbf{T}$-algebras.

Definition 4.1. A pseudovariety of $\mathbf{T}$-algebras is a class of finite $\mathbf{T}$-algebras closed under finite products, subalgebras and quotients.

Notation 4.2. Recall from Remark 2.9 the forgetful functor $|-|: \mathscr{D} \rightarrow$ Set and its left adjoint $X \mapsto \Phi_{X}$. For any finite $\mathbf{T}$-algebra $(A, \alpha)$ to interpret variables from a finite set $X$ in $A$ means to give a morphism $h_{0}: \Phi_{X} \rightarrow A$ in $\mathscr{D}$, or equivalently a T-homomorphism $h:\left(T \Phi_{X}, \mu_{\Phi_{X}}\right) \rightarrow(A, \alpha)$. The corresponding $\widehat{\mathbf{T}}$-homomorphism is denoted $h^{+}: \hat{T} \hat{\Phi_{X}} \rightarrow A$, see Remark 3.4 and 3.9

Definition 4.3. 1. Let $\mathscr{D}$ be a variety of unordered algebras. By a profinite equation over a finite set $X$ of variables is meant a pair $u, v \in \hat{T} \hat{\Phi_{X}}$, denoted $u=v$. A finite $\mathbf{T}$-algebra $(A, \alpha)$ satisfies $u=v$ provided that

$$
h^{+}(u)=h^{+}(v) \text { for all T-homomorphisms } h: T \Phi_{X} \rightarrow A .
$$

2. Let $\mathscr{D}$ be a variety of ordered algebras. A profinite inequation over a finite set $X$ of variables is again a pair $u, v \in \hat{T} \hat{\Phi_{X}}$, denoted $u \leq v$. A finite $\mathbf{T}$-algebra $(A, \alpha)$ satisfies $u \leq v$ provided that

$$
h^{+}(u) \leq h^{+}(v) \text { for all T-homomorphisms } h: T \Phi_{X} \rightarrow A \text {. }
$$

A class $E$ of profinite (in-)equations presents the class of all finite $\mathbf{T}$-algebras that satisfy all (in-)equations in $E$.

Lemma 4.4. Every class of finite $\mathbf{T}$-algebras presented by profinite (in-)equations forms a pseudovariety.

The proof is an easy verification. In the following subsection we show the converse of the lemma: every pseudovariety is presented by profinite equations. 


\subsection{Reiterman's Theorem for T-algebras}

The concept of profinite (in-)equation as introduced above only considers the free finitely generated objects $\Phi_{X}$ of $\mathscr{D}$ as objects of variables. A natural variation is to admit any finite object $X \in \mathscr{D}_{f}$ as an object of variables. That is, we define a profinite equation over $X$ as a pair $u, v \in \hat{T} \hat{X}$, and say that a finite T-algebra $(A, \alpha)$ satisfies $u=v$ if for every T-homomorphism $h:\left(T X, \mu_{X}\right) \rightarrow(A, \alpha)$ the $\widehat{\mathbf{T}}$ homomorphism $h^{+}: \hat{T} \hat{X} \rightarrow A$ merges $u, v$; analogously for inequations. A class of finite $\mathbf{T}$-algebras presented by such profinite equations is still closed under finite products and subalgebras, but not necessarily under quotients. However, it is closed under $U$-split quotients for the forgetful functor $U: \mathscr{D}_{f}^{\mathbf{T}} \rightarrow \mathscr{D}_{f}$, where a surjective morphism $e$ in $\mathscr{D}_{f}^{\mathbf{T}}$ is called $U$-split if there is a morphism $m$ in $\mathscr{D}_{f}$ with $U e \cdot m=i d$.

More generally, we introduce below for a class $\mathscr{X}$ of objects in $\mathscr{D}$ the concept of profinite (in-)equation over $\mathscr{X}$ : a pair of elements of $\hat{T} \hat{X}$ with $X \in \mathscr{X}$. This subsumes both of the above situations: by taking as $\mathscr{X}$ all free finitely generated objects of $\mathscr{D}$ we recover the concept of Section 4.1. And the choice $\mathscr{X}=\mathscr{D}_{f}$ leads to a new variant of Reiterman's theorem: a characterisation of classes of finite T-algebras closed under finite products, subalgebras and $U$-split quotients. The latter can be understood as a finite analogue of Barr's result [9, which states that classes of $\mathbf{T}$-algebras closed under products, subalgebras and $U$-split quotients are in bijective correspondence with quotient monads of $\mathbf{T}$.

Notation 4.5. For a class $\mathscr{X}$ of objects in $\mathscr{D}$ we denote by $\mathcal{E}_{\mathscr{X}}$ the class of all surjective morphisms $e: A \rightarrow B$ with finite codomain such that all objects $X$ of $\mathscr{X}$ are projective w.r.t. $e$. That is, every morphism $f: X \rightarrow B$ factors through $e$.

Assumption 4.6. We assume that a class $\mathscr{X}$ of objects in $\mathscr{D}$ is given that forms a projective presentation of $\mathscr{D}_{f}$, i.e. for every finite object $A \in \mathscr{D}_{f}$ there exists an object $X \in \mathscr{X}$ and a quotient $e: X \rightarrow A$ in $\mathcal{E}_{\mathscr{X}}$.

Definition 4.7. An $\mathscr{X}$-pseudovariety of $\mathbf{T}$-algebras is a class of finite $\mathbf{T}$-algebras closed under finite products, subalgebras and $\mathcal{E}_{\mathscr{X}}$-quotients, i.e. quotients carried by a morphism in $\mathcal{E}_{\mathscr{X}}$.

Example 4.8. (a) For the choice of Section 4.1,

$$
\mathscr{X}=\text { free finitely generated objects of } \mathscr{D},
$$

the class $\mathcal{E}_{\mathscr{X}}$ consists of all surjective morphisms with finite codomain, see Remark 2.9. (c). Clearly Assumption 4.6 is fulfilled since every finite object in a variety $\mathscr{D}$ is a quotient of a free finitely generated one. Thus an $\mathscr{X}$ pseudovariety is simply a pseudovariety in the sense of Definition 4.1 .

(b) If we choose

$$
\mathscr{X}=\mathscr{D}_{f}
$$

then $\mathcal{E}_{\mathscr{X}}$ consists precisely of the split surjections with finite codomain. Indeed, clearly every split surjection lies in $\mathcal{E}_{\mathscr{X}}$. Conversely, given $e: A \rightarrow B$ 
in $\mathcal{E}_{\mathscr{X}}$, apply the definition of $\mathcal{E}_{\mathscr{X}}$ to $X=B$ and $f=i d$. Assumption 4.6 is fulfilled because every object in $\mathscr{D}_{f}$ is a split quotient of itself. A $\mathscr{D}_{f}$ pseudovariety is a class of finite $\mathbf{T}$-algebras closed under finite products, subalgebras and $U$-split quotients.

Definition 4.9. 1. Let $\mathscr{D}$ be a variety of unordered algebras. A profinite equation over $\mathscr{X}$ is an expression of the form $u=v$ with $u, v \in \hat{T} \hat{X}$ and $X \in \mathscr{X}$. A finite $\mathbf{T}$-algebra $(A, \alpha)$ satisfies $u=v$ if

$$
h^{+}(u)=h^{+}(v) \text { for all T-homomorphisms } h: T X \rightarrow A .
$$

2. Let $\mathscr{D}$ be a variety of ordered algebras. A profinite inequation over $\mathscr{X}$ is an expression of the form $u \leq v$ with $u, v \in \hat{T} \hat{X}$ and $X \in \mathscr{X}$. A finite $\mathbf{T}$-algebra $(A, \alpha)$ satisfies $u \leq v$ if

$$
h^{+}(u) \leq h^{+}(v) \text { for all T-homomorphisms } h: T X \rightarrow A
$$

A class $E$ of profinite (in-)equations over $\mathscr{X}$ presents the class of all finite T-algebras that satisfy all (in-)equations in $E$.

Remark 4.10. For any full subcategory $\mathcal{V} \subseteq \mathscr{D}_{f}^{\mathbf{T}}$ closed under finite products and subalgebras, the pro- $\mathcal{V}$ monad of $\mathbf{T}$ is the monad $\widehat{\mathbf{T}}_{\mathcal{V}}=\left(\hat{T}_{\mathcal{V}}, \hat{\mu}^{\mathcal{V}}, \hat{\eta}^{\mathcal{V}}\right)$ on $\widehat{\mathscr{D}}$ defined by replacing in Definition 3.2 the functor $U: \mathscr{D}_{f}^{\mathbf{T}} \rightarrow \mathscr{D}_{f}$ by its restriction $U_{\mathcal{V}}: \mathcal{V} \rightarrow \mathscr{D}_{f}$. That is, $\hat{T}_{\mathcal{V}}$ is the right Kan extension of $\hat{J} U_{\mathcal{V}}$ along itself. In analogy to Remark 3.4, one can describe $\hat{T}_{\mathcal{V}} \hat{X}$ with $X \in \mathscr{D}$ as the cofiltered limit of the diagram of all homomorphisms $h:\left(T X, \mu_{X}\right) \rightarrow(A, \alpha)$ with $(A, \alpha) \in \mathcal{V}$. The limit projections are denoted $h_{\mathcal{V}}^{+}: \hat{T}_{\mathcal{V}} \hat{X} \rightarrow A$. The universal property of $\hat{T}_{\mathcal{V}}$ as a right Kan extension yields a monad morphism $\varphi^{\mathcal{V}}: \widehat{\mathbf{T}} \rightarrow \widehat{\mathbf{T}}_{\mathcal{V}}$; its component $\varphi_{\hat{X}}^{\mathcal{V}}$ for $X \in \mathscr{D}$ is the unique $\widehat{\mathscr{D}}$-morphism making the triangle below commute for all $h:\left(T X, \mu_{X}\right) \rightarrow(A, \alpha)$ with $(A, \alpha) \in \mathcal{V}$.

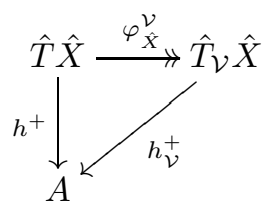

Lemma 4.11. Let $\mathcal{V}$ be a class of finite $\mathbf{T}$-algebras closed under finite products and subalgebras and $u, v \in \hat{T} \hat{X}$ with $X \in \mathscr{D}$.

1. Unordered case: $\varphi_{\hat{X}}^{\mathcal{V}}(u)=\varphi_{\hat{X}}^{\mathcal{V}}(v)$ iff every algebra in $\mathcal{V}$ satisfies $u=v$.

2. Ordered case: $\varphi_{\hat{X}}^{\mathcal{V}}(u) \leq \varphi_{\hat{X}}^{\mathcal{V}}(v)$ iff every algebra in $\mathcal{V}$ satisfies $u \leq v$.

Theorem 4.12 (Reiterman's Theorem for T-algebras). A class of finite $\mathbf{T}$-algebras is an $\mathscr{X}$-pseudovariety iff it is presented by profinite equations over $\mathscr{X}$ (unordered case) resp. profinite inequations over $\mathscr{X}$ (ordered case). 
Proof. Consider first the unordered case. The "if" direction is a straightforward verification. For the "only if" direction let $\mathcal{V}$ be an $\mathscr{X}$-pseudovariety.

(a) In analogy to Proposition 3.5 one can restrict the cofiltered diagram defining $\hat{T}_{\mathcal{V}} \hat{X}$ to surjective homomorphisms $h: T X \rightarrow A$. Then the limit projections $h_{\mathcal{V}}^{+}$and the mediating map $\varphi_{\hat{X}}^{\mathcal{V}}$ in (4.1) are also surjective, see [27, Corollary 1.1.6]. Moreover, since $\varphi^{\mathcal{V}}$ is a monad morphism, the free $\widehat{\mathbf{T}}_{\mathcal{V}}$-algebra $\left(\hat{T}_{\mathcal{V}} \hat{X}, \hat{\mu}_{\hat{X}}^{\mathcal{V}}\right)$ on $\hat{X}$ can be turned into a $\widehat{\mathbf{T}}$-algebra $\left(\hat{T}_{\mathcal{V}} \hat{X}, \hat{\mu}_{\hat{X}}^{\mathcal{V}} \cdot \varphi_{\hat{T}_{\mathcal{V}} \hat{X}}^{\mathcal{V}}\right)$, and $\varphi_{\hat{X}}^{\mathcal{V}}:\left(\hat{T} \hat{X}, \hat{\mu}_{\hat{X}}\right) \rightarrow\left(\hat{T}_{\mathcal{V}} \hat{X}, \hat{\mu}_{\hat{X}}^{\mathcal{V}} \cdot \varphi_{\hat{T}_{\mathcal{V}} \hat{X}}^{\mathcal{V}}\right)$ is a $\widehat{\mathbf{T}}$-homomorphism.

(b) Let $E$ the class of all profinite equations over $\mathscr{X}$ satisfied by all algebras in $\mathcal{V}$. We prove that $\mathcal{V}$ is presented by $E$, which only requires to show that every finite $\mathbf{T}$-algebra $(A, \alpha)$ satisfying all equations in $E$ lies in $\mathcal{V}$.

By Assumption 4.6 choose $X \in \mathscr{X}$ and a quotient $e_{0}: X \rightarrow A$ in $\mathcal{E}_{\mathscr{X}}$, and freely extend $e_{0}$ to a (necessarily surjective) T-homomorphism $e: T X \rightarrow A$. We first show that the corresponding $\widehat{\mathbf{T}}$-homomorphism $e^{+}: \hat{T} \hat{X} \rightarrow \hat{A}$ factors through $\varphi_{\hat{X}}^{\mathcal{V}}$. Indeed, whenever $\varphi_{\hat{X}}^{\mathcal{V}}$ merges $u, v \in \hat{T} \hat{X}$ then the profinite equation $u=v$ lies in $E$ by Lemma 4.11, so $e^{+}$merges $u, v$ since $(A, \alpha)$ satisfies all equations in $E$. Since $\varphi_{\hat{X}}^{\mathcal{V}}$ is surjective by (a), the homomorphism theorem (see Remark 3.8) yields a $\widehat{\mathbf{T}}$-homomorphism $g: \hat{T}_{\mathcal{V}} \hat{X} \rightarrow A$ in $\widehat{\mathscr{D}}$ with $g \cdot \varphi_{\hat{X}}^{\mathcal{V}}=e^{+}$.

(c) By Lemma 3.7(b) the $\widehat{\mathbf{T}}$-homomorphism $g$ factors through the limit cone defining $\widehat{\mathbf{T}}_{\mathcal{V}} \hat{X}$ : there is a T-homomorphism $h: T X \rightarrow B$ with $(B, \beta) \in \mathcal{V}$ and a $\widehat{\mathbf{T}}$-homomorphism $q: B \rightarrow A$ with $q \cdot h_{\mathcal{V}}^{+}=g$. By Proposition 3.10 the morphism $q$ is also a $\mathbf{T}$-homomorphism, and is surjective because $g$ is.

(d) To conclude the proof it suffices to verify that $q$ lies in $\mathcal{E}_{\mathscr{X}}$ (then $(B, \beta) \in \mathcal{V}$ implies $(A, \alpha) \in \mathcal{V}$ because $\mathcal{V}$ is closed under $\mathcal{E}_{\mathscr{X}}$-quotients). Indeed: every morphism $f: Y \rightarrow A$ with $Y \in \mathscr{X}$ factors through $e_{0}$ because $e_{0} \in \mathcal{E}_{\mathscr{X}}$, i.e.

$$
f=e_{0} \cdot k \text { for some } k: Y \rightarrow X \text { in } \mathscr{D} \text {. }
$$

Then the diagram below commutes (for the second triangle see (3.2) ) and shows that $\hat{f}$ factors through $\hat{q}=q$ in $\widehat{\mathscr{D}}$, so $f$ factors through $q$ in $\mathscr{D}$. We conclude that $q \in \mathcal{E}_{\mathscr{X}}$, as desired.

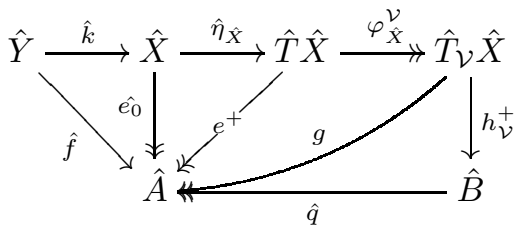

This proves the theorem for the unordered case. The proof for the ordered case is analogous: replace profinite equations by inequations, and use the homomorphism theorem for ordered algebras to construct the morphism $g$. 


\section{$5 \quad$ Applications and Examples}

Let us consider some examples and applications. First note that the original Reiterman theorem and its ordered version emerge from Theorem 4.12 by taking the identity monad $\mathbf{T}=\mathrm{Id}$ and $\mathscr{X}=$ free finitely generated objects of $\mathscr{D}$, see Example 4.8(a). In this case we have $\widehat{\mathbf{T}}=\mathrm{Id}, \mathscr{D}^{\mathbf{T}}=\mathscr{D}, \widehat{\mathscr{D}} \widehat{\mathbf{T}}=\widehat{\mathscr{D}}$, and a profinite equation $u=v$ (resp. a profinite inequation $u \leq v$ ) is a pair $u, v \in \hat{\Phi_{X}}$ for a finite set $X$. We conclude:

Corollary 5.1 (Reiterman [25], Banaschewski [8]). Let $\mathscr{D}$ be a variety of algebras. A class $\mathcal{V} \subseteq \mathscr{D}_{f}$ is a pseudovariety iff it is presented by profinite equations over finite sets of variables.

Corollary 5.2 (Pin and Weil [22]). Let $\mathscr{D}$ be a variety of ordered algebras. A class $\mathcal{V} \subseteq \mathscr{D}_{f}$ is a pseudovariety iff it is presented by profinite inequations over finite sets of variables.

Recall from Isbell [15] that a class $\mathcal{V} \subseteq \mathscr{D}$ is closed under products and subalgebras iff it is presented by implications

$$
\bigwedge_{i \in I} s_{i}=t_{i} \Rightarrow s=t
$$

where $s_{i}, t_{i}, s, t$ are terms and $I$ is a set. Choosing $\mathbf{T}$ to be the identity monad and $\mathscr{X}=\mathscr{D}_{f}$ gives us the counterpart for finite algebras: by Example $4.8(\mathrm{~b})$ a $\mathscr{D}_{f}$-pseudovariety is precisely a class $\mathcal{V} \subseteq \mathscr{D}_{f}$ closed under finite products and subalgebras, since the closure under split quotients is implied by closure under subalgebras. Such a class could be called "quasi-pseudovariety", but to avoid this clumsy terminology we prefer "quasivariety of finite algebras".

Definition 5.3. A quasivariety of finite algebras of $\mathscr{D}$ is a class $\mathcal{V} \subseteq \mathscr{D}_{f}$ closed under finite products and subalgebras.

In analogy to Isbell's result we show that quasivarieties of finite algebras are precisely the classes of finite algebras of $\mathscr{D}$ presented by profinite implications.

Definition 5.4. Let $X$ be a finite set of variables.

1. Unordered case: a profinite implication over $X$ is an expression

$$
\bigwedge_{i \in I} u_{i}=v_{i} \Rightarrow u=v
$$

where $I$ is a set and $u_{i}, v_{i}, u, v \in \hat{\Phi_{X}}$. An object $A \in \mathscr{D}_{f}$ satisfies (5.1) if for every $h: \Phi_{X} \rightarrow A$ with $\hat{h}\left(u_{i}\right)=\hat{h}\left(v_{i}\right)$ for all $i \in I$ one has $\hat{h}(u)=\hat{h}(v)$.

2. Ordered case: a profinite implication over $X$ is an expression

$$
\bigwedge_{i \in I} u_{i} \leq v_{i} \Rightarrow u \leq v
$$

where $I$ is a set and $u_{i}, v_{i}, u, v \in \hat{\Phi_{X}}$. An object $A \in \mathscr{D}_{f}$ satisfies (5.2) if for every $h: \Phi_{X} \rightarrow A$ with $\hat{h}\left(u_{i}\right)=\hat{h}\left(v_{i}\right)$ for all $i \in I$ one has $\hat{h}(u) \leq \hat{h}(v)$. 
A class $P$ of profinite implications presents the class of all finite algebras in $\mathscr{D}$ satisfying all implications in $P$.

Theorem 5.5. For any class $\mathcal{V} \subseteq \mathscr{D}_{f}$ the following statements are equivalent:

1. $\mathcal{V}$ is a quasivariety of finite algebras.

2. $\mathcal{V}$ is presented by profinite (in-)equations over $\mathscr{D}_{f}$.

3. $\mathcal{V}$ is presented by profinite implications.

Proof sketch. $3 \Rightarrow 1$ requires a routine verification, and $1 \Rightarrow 2$ is Theorem 4.12 . For $2 \Rightarrow 3$ assume w.l.o.g. that $\mathcal{V}$ is presented by a single profinite equation $u=v$ with $u, v$ elements of some $X \in \mathscr{D}_{f}$. Express $X$ as a quotient $q: \Phi_{Y} \rightarrow X$ for some finite set $Y$. Let $\left\{\left(u_{i}, v_{i}\right): i \in I\right\}$ be the kernel of $\hat{q}: \hat{\Phi_{Y}} \rightarrow X$ (consisting of all pairs $\left(u_{i}, v_{i}\right) \in \hat{\Phi_{Y}} \times \hat{\Phi_{Y}}$ with $\left.\hat{q}\left(u_{i}\right)=\hat{q}\left(v_{i}\right)\right)$, and choose $u^{\prime}, v^{\prime} \in \hat{\Phi_{Y}}$ with $\hat{q}\left(u^{\prime}\right)=u$ and $\hat{q}\left(v^{\prime}\right)=v$. Then a finite object $A \in \mathscr{D}_{f}$ satisfies the profinite equation $u=v$ iff it satisfies the profinite implication

$$
\bigwedge_{i \in I} u_{i}=v_{i} \Rightarrow u^{\prime}=v^{\prime},
$$

which proves that $\mathcal{V}$ is presented (5.3). Analogously for the ordered case.

Example 5.6. 1. Let $\mathcal{V} \subseteq \mathbf{M o n}_{f}$ be the quasivariety of all finite monoids whose only invertible element is the unit. It is presented by the profinite implication $x^{\omega}=1 \Rightarrow x=1$ over the set of variables $X=\{x\}$. Here the profinite word $x^{\omega} \in \widehat{X^{*}}$ is interpreted, for every finite monoid $M$ with $x$ interpreted as $m \in M$, as the unique idempotent power of $m$. Indeed, if $M$ has no nontrivial invertible elements, it satisfies the implication: given $m \neq 1$ and $m^{k}$ idempotent, then $m^{k} \neq 1$ (otherwise $m$ has the inverse $m^{k-1}$ ). Conversely, if $M$ satisfies the implication and $m$ is invertible, then so is its idempotent power $m^{k}$. Hence $m^{k} \cdot m^{k}=m^{k}$ implies $m^{k}=1$, so $m=1$.

2. Let Pos be the variety of posets (i.e. the variety of all ordered algebras over the empty signature). The quasivariety $\mathcal{V} \subseteq \mathbf{P o s}_{f}$ of finite discrete posets is presented by the profinite implication $v \leq u \Rightarrow u \leq v$ over the set $X=\{u, v\}$.

Remark 5.7. As indicated before all concepts in this paper also apply to a setting where $\mathscr{D}$ is a many-sorted variety of algebras or ordered algebras. In this case an algebra is finite if the disjoint union of the underlying sets of all sorts is a finite set. By a profinite equation over $X \in \mathscr{D}$ is a meant pair of elements $u, v$ in some sort $s$ of $\hat{T} \hat{X}$, and it is satisfied by a finite $\mathbf{T}$-algebra $A$ if for every T-homomorphism $h: T X \rightarrow A$ the $s$-component of $h^{+}: \hat{T} \hat{X} \rightarrow A$ merges $u, v$. Similarly for profinite inequations and profinite implications.

Example 5.8. Consider the variety $\mathscr{D}$ of directed graphs, i.e. algebras for the two-sorted signature consisting of a sort Ob (objects), a sort Mor (morphisms) and two unary operations $s, t$ : Mor $\rightarrow$ Ob specifying the source and target of 
a morphism. Then Cat, the category of small categories and functors, is isomorphic to $\mathscr{D}^{\mathbf{T}}$ for the monad $\mathbf{T}$ constructing the free category on a graph. Choosing $\mathscr{X}=$ free finitely generated graphs, Theorem 4.12 shows that every pseudovariety of categories, i.e. every class of finite categories closed under finite products, subcategories (represented by injective functors) and quotient categories (represented by surjective functors), can be specified by profinite equations over a two-sorted set of variables. This result was essentially proved by Jones [17. The difference is that he restricts to quotients represented by surjective functors which are bijective on objects, and replaces subcategories by faithful functors. Moreover, profinite equations are restricted to the sort of morphisms.

\section{Conclusions and Future Work}

Motivated by recent developments in algebraic language theory, we generalised Reiterman's theorem to finite algebras for an arbitrary monad $\mathbf{T}$ on a base category $\mathscr{D}$. Here $\mathscr{D}$ is a variety of (possibly ordered, many-sorted) algebras. The core concept of our paper is the profinite monad $\widehat{\mathbf{T}}$ of $\mathbf{T}$, which makes it possible to introduce profinite (in-)equations at the level of monads and prove that they precisely present pseudovarieties of $\mathbf{T}$-algebras.

Referring to the diagram in the Introduction, our Reiterman theorem is presented in a setting that unifies the two categorical approaches to algebraic language theory of Bojańzcyk [11] and in our work [2,4,12. The next step is to also derive an Eilenberg theorem in this setting. For each monad $\mathbf{T}$ on a category of sorted sets, Bojańczyk [1] proved an Eilenberg-type characterisation of pseudovarieties of $\mathbf{T}$-algebras: they correspond to varieties of $\mathbf{T}$-recognisable languages. Here by a "language" is meant a function from $T X$ to $\{0,1\}$ for some alphabet $X$, and a variety of languages is a class of such languages closed under boolean operations, homomorphic preimages and a suitably generalised notion of derivatives. On the other hand, as indicated in Example 2.12, one needs to consider monoids on algebraic categories beyond Set in order to study varieties of languages with relaxed closure properties, e.g. dropping closure under complement or intersection. The aim is thus to prove an Eilenberg theorem parametric in a monad $\mathbf{T}$ on an algebraic category $\mathscr{D}$. Observing that e.g. for $\mathscr{D}=$ Set the monad $\widehat{\mathbf{T}}$ on Stone dualises to a comonad on the category of boolean algebras, we expect this can be achieved in a duality-based setting along the lines of Gehrke, Grigorieff and Pin [14] and our work [2,4].

Throughout this paper we presented the case of ordered and unordered algebras as separated but analogous developments. Pin and Weil 22. gave a uniform treatment of ordered and unordered algebras by generalising Reiterman's theorem from finite algebras to finite first-order structures. A similar approach should also work in our categorical framework: replace $\mathscr{D}$ by a variety of relational algebras over a quasivariety $\mathcal{Q}$ of relational first-order structures, with $\mathcal{Q}=$ Set and $\mathcal{Q}=$ Pos covering the case of algebras and ordered algebras.

Finally, observe that categories of the form $\mathscr{D}^{\mathbf{T}}$, where $\mathscr{D}$ is a many-sorted variety of algebras and $\mathbf{T}$ is an accessible monad, correspond precisely to locally 
presentable categories. This opens the door towards an abstract treatment, and further generalisation, of Reiterman's theorem in purely categorical terms.

\section{References}

1. Adámek, J., Rosický, J., Vitale, E.: Algebraic Theories. Cambridge University Press (2011)

2. Adámek, J., Milius, S., Myers, R.S.R., Urbat, H.: Generalized Eilenberg theorem I : Local varieties of languages. In: Muscholl, A. (ed.) Proc. FoSSaCS'14. LNCS, vol. 8412, pp. 366-380. Springer Berlin Heidelberg (2014)

3. Adámek, J., Milius, S., Urbat, H.: Syntactic monoids in a category. In: Moss, L.S., Sobocinski, P. (eds.) Proc. CALCO'15 (2015)

4. Adámek, J., Myers, R.S.R., Urbat, H., Milius, S.: Varieties of languages in a category. In: Proc. LICS'15. IEEE (2015)

5. Adámek, J., Porst, H.E.: On tree coalgebras and coalgebra presentations. Theor. Comput. Sci. 311(1-3), 257-283 (2004)

6. Adámek, J., Rosický, J.: Locally Presentable and Accessible Categories. Cambridge University Press (1994)

7. Almeida, J.: On pseudovarieties, varieties of languages, filters of congruences, pseudoidentities and related topics. Algebra universalis 27(3), 333-350 (1990)

8. Banaschewski, B.: The Birkhoff Theorem for varieties of finite algebras. Algebra universalis 17(1), 360-368 (1983)

9. Barr, M.: HSP subcategories of Eilenberg-Moore algebras. Theory Appl. Categ. 10(18), 461-468 (2002)

10. Bloom, S.L.: Varieties of ordered algebras. J. Comput. Syst. Sci. 13(2), 200-212 (1976)

11. Bojańczyk, M.: Recognisable languages over monads. In: Potapov, I. (ed.) Proc. DLT, LNCS, vol. 9168, pp. 1-13. Springer (2015), full version: http://arxiv.org/abs/1502.04898

12. Chen, L.T., Urbat, H.: A fibrational approach to automata theory. In: Moss, L.S., Sobocinski, P. (eds.) Proc. CALCO'15 (2015)

13. Eilenberg, S.: Automata, Languages, and Machines, vol. 2. Academic Press, New York (1976)

14. Gehrke, M., Grigorieff, S., Pin, J.E.: Duality and equational theory of regular languages. In: Aceto, L., Damgå rd, I., Goldberg, L.A., Halldórsson, M.M., Ingólfsdóttir, A., Walukiewicz, I. (eds.) Proc. ICALP'08, Part II, LNCS, vol. 5126, pp. 246-257. Springer Berlin Heidelberg (2008)

15. Isbell, J.R.: Subobjects, adequacy, completeness and categories of algebras. Instytut Matematyczny Polskiej Akademi Nauk (1964)

16. Johnstone, P.T.: Stone spaces. Cambridge University Press (1982)

17. Jones, P.R.: Profinite categories, implicit operations and pseudovarieties of categories. J. Pure Appl. Algebr. 109(1), 61-95 (1996)

18. Kennison, J.F., Gildenhuys, D.: Equational completion, model induced triples and pro-objects. J. Pure Appl. Algebr. 1(4), 317-346 (1971)

19. Linton, F.E.J.: An outline of functorial semantics. In: Eckmann, B. (ed.) Semin. Triples Categ. Homol. Theory, LNM, vol. 80, pp. 7-52. Springer Berlin Heidelberg (1969)

20. Mac Lane, S.: Categories for the working mathematician. Springer, 2 edn. (1998) 
21. Pin, J.E.: A variety theorem without complementation. Russ. Math. (Izvestija vuzov.Matematika) 39, 80-90 (1995)

22. Pin, J.E., Weil, P.: A Reiterman theorem for pseudovarieties of finite first-order structures. Algebra Universalis 35, 577-595 (1996)

23. Polák, L.: Syntactic semiring of a language. In: Sgall, J., Pultr, A., Kolman, P. (eds.) Proc. MFCS. LNCS, vol. 2136, pp. 611-620. Springer (2001)

24. Priestley, H.A.: Ordered topological spaces and the representation of distributive lattices. Proc. London Math. Soc. 3(3), 507 (1972)

25. Reiterman, J.: The Birkhoff theorem for finite algebras. Algebra Universalis 14(1), 1-10 (1982)

26. Reutenauer, C.: Séries formelles et algèbres syntactiques. J. Algebra 66, 448-483 (1980)

27. Ribes, L., Zalesskii, P.: Profinite Groups. A Series of Modern Surveys in Mathematics, Springer Berlin Heidelberg (2010)

28. Schützenberger, M.P.: On finite monoids having only trivial subgroups. Inform. and Control 8, 190-194 (1965)

29. Street, R.: The formal theory of monads. J. Pure Appl. Algebr. 2(2), 149-168 (1972)

30. Wilke, T.: An Eilenberg theorem for infinity-languages. In: Proc. ICALP'91. pp. 588-599 (1991)

\section{A Topological toolkit}

The proofs of the following lemmas can be found in Chapter 1 of [27].

Lemma A.1. Let $\tau: D_{1} \rightarrow D_{2}$ be a natural transformation between cofiltered diagrams (of the same index) in the category of compact Hausdorff spaces. If each $\tau_{i}: D_{1} i \rightarrow D_{2} i$ is surjective, so is the mediating map

$$
\operatorname{Lim} \tau: \operatorname{Lim} D_{1} \rightarrow \operatorname{Lim} D_{2}
$$

In particular, if $\tau_{i}: X \rightarrow D i$ is a cone of surjections, then the mediating map $h=\lim \tau$ is surjective.

Lemma A.2. Let $\left(X \stackrel{\rho_{i}}{\longrightarrow} X_{i}\right)_{i}$ be a limit of a cofiltered diagram in the category of topological spaces, and $\left(Y \stackrel{f_{i}}{\longrightarrow} X_{i}\right)_{i}$ a cone of that diagram consisting of surjective continuous functions. Then, the mediating map

$$
f: Y \rightarrow X
$$

is dense, i.e. the image $f[Y]$ is a dense subset of $X$.

Lemma A.3. Let $D$ be a cofiltered diagram in the category of compact Hausdorff spaces. If all $D(i \stackrel{f}{\rightarrow} j)$ are surjective, so is each projection $\rho_{i}: \operatorname{Lim} D \rightarrow$ Di. 


\section{B Detailed proofs}

This appendix contains all proofs and additional details we omitted due to space restrictions.

Definition B.1. An object $A$ in a category $\mathscr{A}$ is called finitely copresentable if the hom-functor $\mathscr{A}(-, A): \mathscr{A}^{\text {op }} \rightarrow$ Set preserves filtered colimits. Equivalently, given a cofiltered limit cone $\pi_{i}: B \rightarrow B_{i}(i \in I)$ in $\mathscr{A}$, any morphism $f: B \rightarrow A$ factors essentially uniquely through some $\pi_{i}$. The category $\mathscr{A}$ is locally finitely copresentable if (i) $\mathscr{A}$ is complete, (ii) $\mathscr{A}$ has only a set of finitely copresentable objects up to isomorphism, and (iii) every object of $A$ is a cofiltered limit of finitely copresentable objects.

Remark B.2. For any small category $\mathscr{A}_{0}$ with finite limits the pro-completion $\mathscr{A}=$ Pro- $\mathscr{A}_{0}$, i.e. the free completion of $\mathscr{A}_{0}$ under cofiltered limits, is locally finitely copresentable, and the full subcategory of finitely copresentable objects is equivalent to $\mathscr{A}_{0}$. See e.g. [1, Theorem 6.23].

Details for Remark [2.5, By [16, Remark VI.2.4] the category $\widehat{\mathscr{D}}$ is the procompletion of $\mathscr{D}_{f}$. (The argument given there is for varieties of single-sorted algebras, but also applies to the ordered and many-sorted case.) Hence $\widehat{\mathscr{D}}$ is locally finitely copresentable and the objects of $\mathscr{D}_{f}$ are precisely the finitely copresentable objects of $\widehat{\mathscr{D}}$, see Remark B.2. This implies (ii). For (i) see [6, Proposition 1.22].

Lemma B.3. $\widehat{\mathscr{D}}$ is closed under cofiltered limits in the category of topological $\mathscr{D}$-algebras. In particular, the forgetful functor $V: \widehat{\mathscr{D}} \rightarrow \mathscr{D}$ preserves cofiltered limits.

Proof. Let $\operatorname{Top}(\mathscr{D})$ denote the category of topological $\mathscr{D}$-algebras. Since $\hat{J}$ : $\mathscr{D}_{f} \longmapsto \widehat{\mathscr{D}}$ is the pro-completion of $\mathscr{D}_{f}$ the inclusion functor $I: \mathscr{D}_{f} \longmapsto \operatorname{Top}(\mathscr{D})$ has an essentially unique extension $I^{\prime}: \widehat{\mathscr{D}} \rightarrow \mathbf{T o p}(\mathscr{D})$ preserving cofiltered limits. The functor $I^{\prime}$ maps an object $D \in \widehat{\mathscr{D}}$ to the cofiltered limit $I^{\prime} D$ of the diagram of all morphisms $f: D \rightarrow D^{\prime}$ with finite codomain. But since $D$ is profinite it follows $I^{\prime} D \cong D$, that is, $I^{\prime}$ is just the inclusion.

That $V$ preserves cofiltered limits now follows from the fact that limits in $\operatorname{Top}(\mathscr{D})$ are formed on the level of $\mathscr{D}$.

Remark B.4. Since $\widehat{\mathscr{D}}$ is locally finitely copresentable so is the arrow category $\widehat{\mathscr{D}} \rightarrow$. Its finitely copresentable objects are precisely the morphisms in $\mathscr{D}_{f}$, i.e. morphisms with finite domain and codomain, see [6. Corollary 1.54 and Example 1.55]. Hence every morphism $f: A \rightarrow B$ in $\widehat{\mathscr{D}}$ is a cofiltered limit (taken in $\widehat{\mathscr{D}} \rightarrow$ ) of morphisms in $\mathscr{D}_{f}$. 
Proof (Lemma 2.6). Let $f: A \rightarrow B$ in $\widehat{\mathscr{D}}$. Express $f$ as a cofiltered limit of morphisms $f_{i}$ in $\mathscr{D}_{f}$ with the limit cone $\left(a_{i}, b_{i}\right): f \rightarrow f_{i}$, see Remark B.4. Factorise $f_{i}=m_{i} \cdot e_{i}$ into a quotient followed by a subalgebra in $\mathscr{D}$.

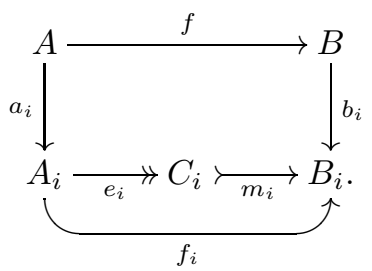

Due to the diagonal fill-in property, the finite objects $C_{i}$ form a cofiltered diagram with $\left(e_{i}\right)$ and $\left(m_{i}\right)$ natural transformations. Let $\left(c_{i}: C \rightarrow C_{i}\right)$ be its limit in $\widehat{\mathscr{D}}$, and, $e:=\operatorname{Lim} e_{i}$, and $m:=\operatorname{Lim} m_{i}$ in $\widehat{\mathscr{D}} \rightarrow$, as shown in the diagram below:

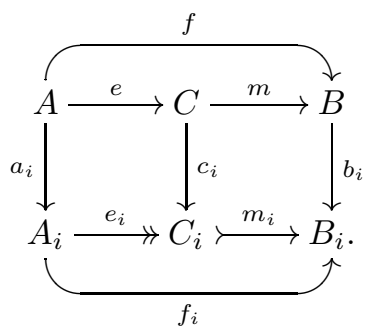

The morphism $e$ is surjective by Lemma A.1. To show that $m$ is injective observe that $m(c)=m\left(c^{\prime}\right)$ implies

$$
m_{i} \cdot c_{i}(c)=b_{i} \cdot m(c)=b_{i} \cdot m\left(c^{\prime}\right)=m_{i} \cdot c_{i}\left(c^{\prime}\right)
$$

thus $c=c^{\prime}$ since $m_{i}$ is monic and the morphisms $c_{i}$ are jointly monic. In the ordered case, to show that $m$ is order-reflecting use instead that $m_{i}$ is orderreflecting and the morphisms $c_{i}$ are jointly order-reflecting.

Proof (Proposition 2.8). Since $V$ preserves cofiltered limits by LemmaB.3 there is a unique mediating morphism $\eta_{D}: D \rightarrow V \hat{D}$ in $\mathscr{D}$ with $V \hat{h} \cdot \eta_{D}=h$ for all $h: D \rightarrow D^{\prime}$ with $D^{\prime} \in \mathscr{D}_{f}$. We need to show that for any $g: D \rightarrow V E$ with $E \in \widehat{\mathscr{D}}$ there is a unique $g^{\circledR}: \hat{D} \rightarrow E$ with $V g^{\circledR} \cdot \eta_{D}=g$.

Express $E$ as a cofiltered limit $p_{i}: E \rightarrow E_{i}, i \in I$, with $E_{i} \in \mathscr{D}_{f}$. Then the morphisms $\widehat{V p_{i} \cdot g}: \hat{D} \rightarrow E_{i}$ form a cone, so there is a unique $g^{\circledR}: \hat{D} \rightarrow E$ with $p_{i} \cdot g^{\Theta}=\widehat{V p_{i} \cdot g}$ for all $i$. It follows that

$$
V p_{i} \cdot V g^{@} \cdot \eta_{D}=V\left(\widehat{V p_{i} \cdot g}\right) \cdot \eta_{D}=V p_{i} \cdot g .
$$

Since $V$ preserves cofiltered limits the morphisms $V p_{i}$ are jointly monic, so $V g^{\circledR} \cdot \eta_{D}=g$. Moreover, since one can restrict the limit cone defining $\hat{D}$ to surjective morphisms $h: D \rightarrow D^{\prime}$ (they form an initial subdiagram of $D \downarrow \mathscr{D}_{f}$ ), 
the morphism $\eta_{D}$ is dense by Lemma A.2 (where $D$ is viewed as a discrete topological space). This implies that the morphism $g^{@}$ is uniquely determined by the equation $V g^{@} \cdot \eta_{D}=g$, as desired.

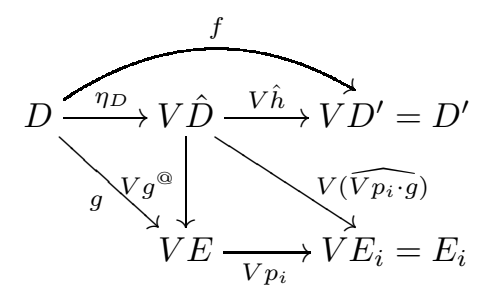

We conclude that $V$ has a left adjoint whose action on objects is given by $D \mapsto \hat{D}$, and on morphisms $h: D \rightarrow E$ by $h \mapsto\left(\eta_{E} \cdot h\right)^{@}$. It remains to show that for $E$ finite we have $\left(\eta_{E} \cdot g\right)^{@}=\hat{g}$. Indeed, in this case we have $\hat{E}=E$ and $\eta_{E}=i d$, and the limit cone $\left(p_{i}\right)$ can be chosen trivial (that is, $I=\{1\}, E_{1}=E$ and $\left.p_{1}=i d\right)$. Then

$$
\left(g \eta_{E}\right)^{@}=g^{@}=p_{1} \cdot g^{@}=\widehat{V p_{1} \cdot g}=\hat{g} .
$$

Details for Remark 2.1]. We prove the claim that the factorisation system of $\mathscr{D}$ of lifts to $\mathscr{D}^{\mathbf{T}}$. Letting $h=m \cdot e$ be the canonical factorisation of $h$ in $\mathscr{D}$, diagonal fill-in gives a unique $\gamma: T C \rightarrow C$ making the diagram below commute. One easily verifies that $(C, \gamma)$ is a $\mathbf{T}$-algebra, so $e:(A, \alpha) \rightarrow(C, \gamma)$ and $m:(C, \gamma) \longmapsto(B, \beta)$ are $\mathbf{T}$-homomorphisms.

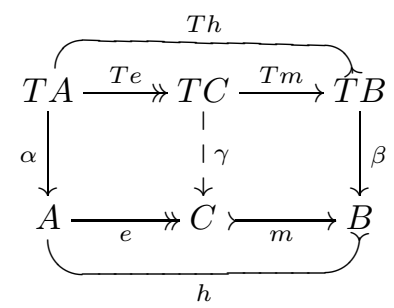

Notation B.5. Recall from Remark 3.4 that for $X \in \widehat{\mathscr{D}}$ the object $\hat{T} X$ is the limit of the diagram

$$
(X \downarrow \hat{J} U) \rightarrow \widehat{\mathscr{D}}, \quad(f: X \rightarrow \hat{J} U(A, \alpha)) \mapsto A .
$$

We denote the limit projections by $f^{*}: \hat{T} X \rightarrow A$.

Details for Remark 3.4. We show that the diagrams (3.2) commute for all $h$ : $\left(T D, \mu_{D}\right) \rightarrow(A, \alpha)$ with $(A, \alpha) \in \mathscr{D}_{f}^{\mathbf{T}}$. As for the left hand diagram, recall the definition of $\hat{\eta}=\left(i d_{\hat{J} U}\right)^{\dagger}$ in the limit formula for right Kan extensions: for each $f: X \rightarrow \hat{J} U(A, \alpha)$, the collection of $f$ 's forms a compatible cone for the limit 
defining $\hat{T} X$, and $\hat{\eta}_{X}$ is the unique mediating morphism satisfying the diagram

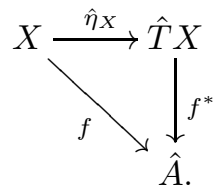

For $X=\hat{D}$ and $f=\widehat{h \eta_{D}}$ we have $f^{*}=h^{+}$, which proves the claim. Similarly for the right hand diagram.

Proof (Proposition 3.5). By Remark 2.11, we can factorise every morphism $h:\left(T D, \mu_{D}\right) \rightarrow$ $(A, \alpha)$ as a quotient followed by a subalgebra:

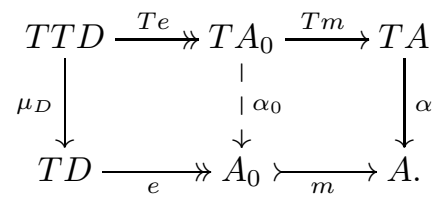

This shows the subdiagram of all quotients of $\left(T D, \mu_{D}\right)$ is initial in the diagram defining $\hat{T} \hat{D}$. Hence their limits are the same.

Proof. By Proposition 3.5 we have the limit cone $e^{+}: \hat{T} \hat{D} \rightarrow A$ in $\widehat{\mathscr{D}}$. That all $e^{+}$'s are surjective follows by Lemma A.3. Further, the morphisms $e^{+}$are $\widehat{\mathbf{T}}$-algebra homomorphisms and hence $e^{+}:\left(\hat{T} X, \hat{\mu}_{X}\right) \rightarrow\left(A, \alpha^{+}\right)$is a cofiltered limit cone in $\widehat{\mathscr{D}} \widehat{\mathbf{T}}$ by (3.2), , Remark 3.9 and the fact that limits of $\widehat{\mathbf{T}}$-algebras are formed on the level of $\widehat{\mathscr{D}}$.

Proof (Lemma 3.7). (a) $\hat{T}$ preserves cofiltered limits: for the purpose of this proof it is convient to express the limit defining $\hat{T} X$ for $X \in \widehat{\mathscr{D}}$, see Notation B.5, by the end formula

$$
\hat{T} X=\int_{(A, \alpha) \in \mathscr{D}_{f}^{\mathrm{T}}} \widehat{\mathscr{D}}(X, \hat{A}) \pitchfork \hat{A} .
$$

Note that the power functor $-\pitchfork B: \mathbf{S e t}^{\mathrm{op}} \rightarrow \widehat{\mathscr{D}}$ preserves limits, and for $A$ finite the hom-functor $\widehat{\mathscr{D}}(-, \hat{A}): \widehat{\mathscr{D}}^{\mathrm{op}} \rightarrow$ Set preserves filtered colimits. Hence the composite $\widehat{\mathscr{D}}(-, \hat{A}) \pitchfork B: \widehat{\mathscr{D}} \rightarrow \widehat{\mathscr{D}}$ preserves cofiltered limits. Expressing $\hat{T}$ as an end, it follows that $\hat{T}$ preserves cofiltered limits:

$$
\begin{aligned}
\hat{T}\left(\operatorname{Lim} X_{i}\right) & =\int_{(A, \alpha)} \widehat{\mathscr{D}}\left(\operatorname{Lim} X_{i}, \hat{A}\right) \pitchfork \hat{A} \\
& =\int_{(A, \alpha)} \operatorname{Lim}_{i}\left(\widehat{\mathscr{D}}\left(X_{i}, \hat{A}\right) \pitchfork \hat{A}\right) \\
& =\operatorname{Lim}_{i}\left(\int_{(A, \alpha)} \widehat{\mathscr{D}}\left(X_{i}, \hat{A}\right) \pitchfork \hat{A}\right) \\
& =\operatorname{Lim}_{i}\left(\hat{T} X_{i}\right) .
\end{aligned}
$$


$\hat{T}$ preserves surjective morphisms: let $f: X \rightarrow Y$ be a surjective morphism of $\widehat{\mathscr{D}}$. We need to show that $\hat{T} f$ is surjective.

(1) Suppose first that $X$ and $Y$ are finite. Then, $f=\widehat{f}_{0}$ for some $f_{0}: X_{0} \rightarrow$ $Y_{0}$ in $\mathscr{D}$. Since $T$ preserves surjections by Assumption 3.1, the morphism $T f_{0}: T X_{0} \rightarrow T Y_{0}$ is surjective, so every quotient $e$ of the free $\mathbf{T}$-algebra $\left(T Y_{0}, \mu_{Y_{0}}\right)$ in $\mathscr{D}^{\mathbf{T}}$ yields a quotient $\bar{e}:=e \cdot T f_{0}$ of $\left(T X_{0}, \mu_{X_{0}}\right)$. Due to Proposition 3.5, $\hat{T} f$ is the mediating morphism

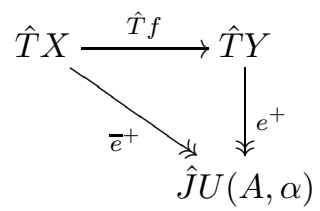

for all quotients $e$ of $\left(T Y_{0}, \mu_{Y_{0}}\right)$. Each component $\bar{e}^{+}$is surjective by Lemma A.3, so the mediating morphism $\hat{T} f$ is also surjective by Lemma A.1.

(2) Now let $X$ and $Y$ be arbitrary. By Remark B.4 the morphism $f$ is the cofiltered limit in $\widehat{\mathscr{D}} \rightarrow$ of all morphisms $\left(x_{i}, y_{i}\right): f \rightarrow f_{i}$ with $f_{i}: X_{i} \rightarrow Y_{i}$ in $\mathscr{D}_{f}$. Let $f_{i}=e_{i} \cdot m_{i}$ be the canonical factorisation of $f_{i}$ in $\widehat{\mathscr{D}}$.

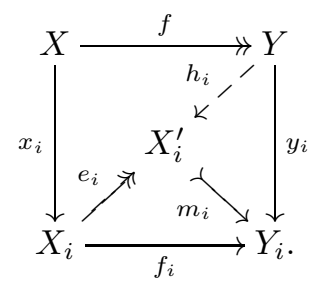

There exists a unique morphism $h_{i}: Y \rightarrow X_{i}^{\prime}$ by the diagonal fill-in property. It follows that surjections $\left(e_{i}\right)$ form an initial cofiltered subdiagram. Since $\hat{T}$ preserves cofiltered limits by (a), $\hat{T} f$ is the limit of $\hat{T} e_{i}: \hat{T} X_{i} \rightarrow \hat{T} Y_{i}$. However, each $\hat{T} e_{i}$ is surjective as proved in (1), so by Lemma A.1 it follows that $\hat{T} f$ is surjective.

(c) We need to prove that finite $\widehat{\mathbf{T}}$-algebras are finitely copresentable in $\widehat{\mathscr{D}} \widehat{\mathbf{T}}$ Consider the category $\operatorname{Alg}(\hat{T})$ of algebras for the functor $\hat{T}$. In [5, Lemma $3.2]$ it is proved that for a cofinitary functor on a locally finitely copresentable category, every algebra $(A, \alpha)$ with $A$ finitely copresentable is finitely copresentable in $\operatorname{Alg}(\hat{T})$. Now observe that $\widehat{\mathscr{D}} \widehat{\mathbf{T}}$ is a full subcategory of $\operatorname{Alg}(\hat{T})$ closed under limits, $\widehat{\mathscr{D}}$ is locally finitely copresentable, and finite objects in $\widehat{\mathscr{D}}$ are finitely copresentable, see Remark 2.5 . Hence, finite $\widehat{\mathbf{T}}$-algebras are finitely copresentable in $\widehat{\mathscr{D}}^{\mathbf{T}}$. 
Remark B.6. Every right adjoint preserves right Kan extensions, so $(V \hat{T}, V \varepsilon)$ is the right Kan extension of $V \hat{J} U$ along $\hat{J} U$. Moreover, there is a natural transformation $\gamma: T V \hat{J} U \rightarrow V \hat{J} U$ whose component at $(A, \alpha) \in \mathscr{D}_{f}^{\mathbf{T}}$ is given by $\alpha$ itself:

$$
\gamma_{(A, \alpha)}:=\alpha: T U(A, \alpha) \rightarrow U(A, \alpha) .
$$

Therefore, by the universal property of $V \hat{T}$, there is a unique natural transformation $\varphi: T V \rightarrow V \hat{T}$ such that $\gamma=V \varepsilon \cdot \varphi \hat{J} U$. By the limit formula for right Kan extensions and Remark 3.4 the component $\varphi_{\hat{X}}$ for $X \in \mathscr{D}$ is the unique mediating morphism making the following diagram commute for all $h:\left(T X, \mu_{X}\right) \rightarrow(A, \alpha)$ with $(A, \alpha) \in \mathscr{D}_{f}^{\mathbf{T}}$ :

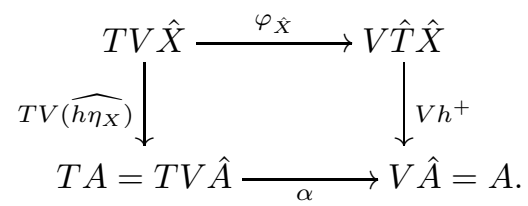

In particular, putting $h=\alpha$ for $(A, \alpha) \in \mathscr{D}_{f}^{\mathbf{T}}$, we get the commutative triangle

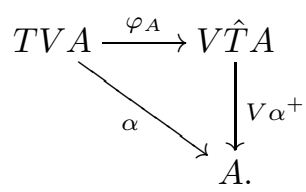

Proposition B.7. (a) $\varphi: T V \rightarrow V \hat{T}$ is a monad morphism [29], i.e. the following diagrams commute:

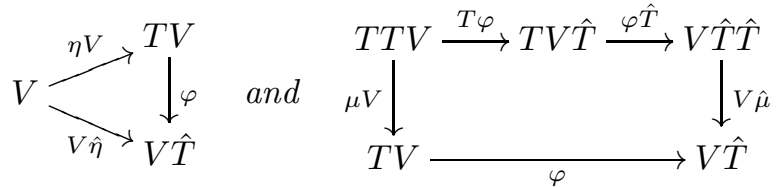

(b) For finite $X$ the morphism $\varphi_{X}: T V X \rightarrow V \hat{T} X$ is dense.

Proof. (a) The following pasting diagrams use the universality of $\varepsilon$ and the monad laws. 
- The preservation of unit $\varphi \cdot \eta V=V \hat{\eta}$ :

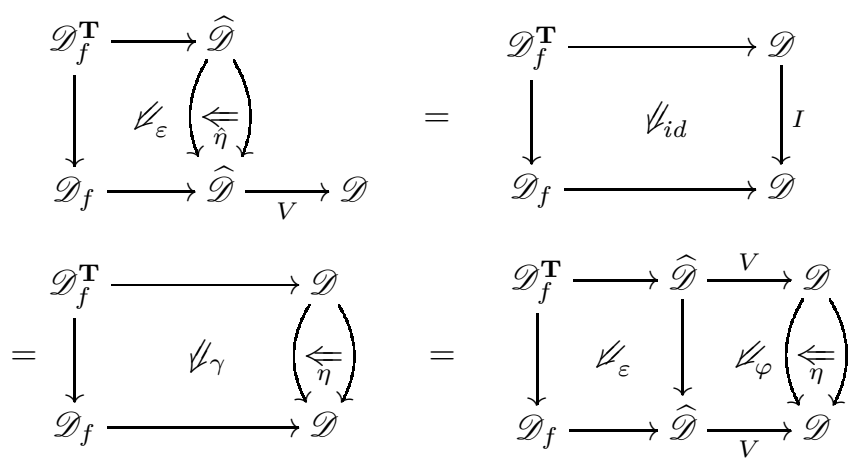

- The preservation of multiplication $\varphi \cdot \mu_{V}=V \hat{\mu} \cdot \varphi \hat{T} \cdot T \varphi$ :
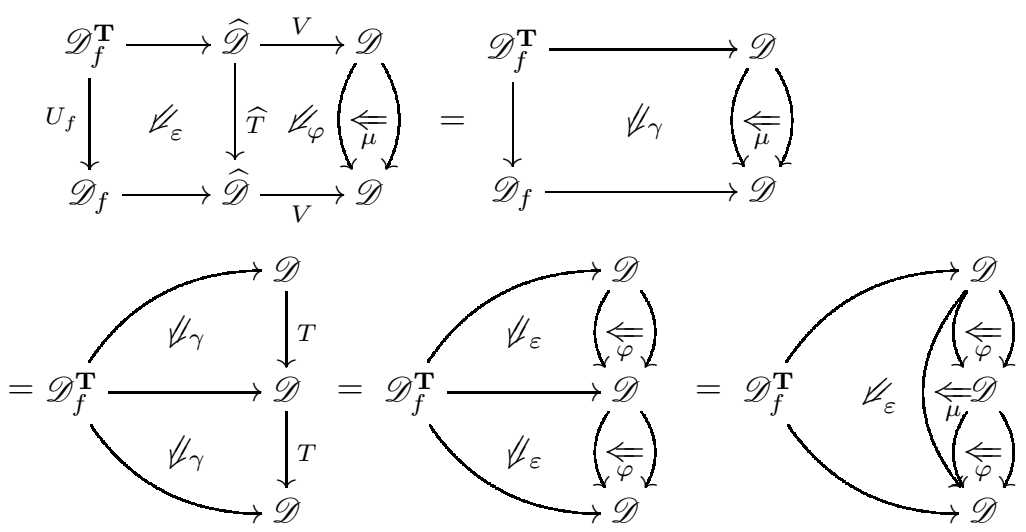

(b) If $X$ is finite we have for all $h:\left(T X, \mu_{X}\right) \rightarrow(A, \alpha)$ with $(A, \alpha) \in \mathscr{D}_{f}^{\mathbf{T}}$ :

$$
\alpha \cdot T V\left(\widehat{h \eta_{X}}\right)=\alpha \cdot T h \cdot T \eta_{X}=h \cdot \mu_{X} \cdot T \eta_{X}=h
$$

using that $h$ is a $\mathbf{T}$-homomorphism and the unit law of the monad T. In particular, if $h$ is surjective, so is the cone $\alpha \cdot T V\left(\widehat{h \eta_{X}}\right)$ in (4.1). By Proposition 3.5 and Lemma A.2 this implies that $\varphi_{X}$ is dense.

Proof (Proposition 3.10). (a) The maps $(A, \alpha) \mapsto\left(A, \alpha^{+}\right)$and $h \mapsto \widehat{h}$ define a functor

$$
K^{\mathbf{T}}: \mathscr{D}_{f}^{\mathbf{T}} \rightarrow \widehat{\mathscr{D}}_{f}^{\widehat{\mathbf{T}}}
$$

To see this we only need to prove that for every T-homomorphism $h:(A, \alpha) \rightarrow$ $(B, \beta)$ is also a $\widehat{\mathbf{T}}$-homomorphism $\left(A, \alpha^{+}\right) \rightarrow\left(B, \beta^{+}\right)$. Indeed, in the diagram below the right hand square commutes when precomposed with $\hat{\eta}_{A}$, so it commutes 
by the universality of $\hat{\eta}_{A}$.

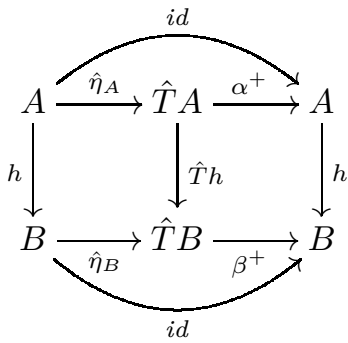

(b) Conversely, the monad morphism $\varphi: T V \rightarrow V \hat{T}$, see Remark B.6 and Proposition B.7, induces a functor

$$
V^{\mathbf{T}}: \widehat{\mathscr{D}}_{f}^{\widehat{\mathbf{T}}} \rightarrow \mathscr{D}_{f}^{\mathbf{T}}
$$

mapping $(A, \alpha)$ to $\left(V A, V \alpha \cdot \varphi_{A}\right)$ and $h:(A, \alpha) \rightarrow(B, \beta)$ to $V h$.

(c) It remains to prove that $K^{\mathbf{T}}$ and $V^{\mathbf{T}}$ are mutually inverse functors. Clearly this holds on morphisms, since both functors are identity on morphisms. As for objects, for every finite $\mathbf{T}$-algebra $(A, \alpha)$ we have

$$
\begin{aligned}
V^{\mathbf{T}} K^{\mathbf{T}}(A, \alpha) & =V^{\mathbf{T}}\left(\hat{A}, \alpha^{+}\right) & & \{\text {by definition }\} \\
& =\left(V \hat{A}, V \alpha^{+} \cdot \varphi_{A}\right) & & \{\text { by definition }\} \\
& =(A, \alpha) . & & \{\text { by (B.2) }\}
\end{aligned}
$$

Conversely, let $(A, \alpha)$ be a finite $\widehat{\mathbf{T}}$-algebra and $\left(A, \alpha^{\prime}\right):=K^{\mathbf{T}} V^{\mathbf{T}}(A, \alpha)$. Applying $V^{\mathbf{T}}$ on both sides, we have $V^{\mathbf{T}}\left(A, \alpha^{\prime}\right)=V^{\mathbf{T}} K^{\mathbf{T}} V^{\mathbf{T}}(A, \alpha)=V^{\mathbf{T}}(A, \alpha)$, using that $V^{\mathbf{T}} K^{\mathbf{T}}=\mathrm{Id}$ as proved above. That is, $V \alpha^{\prime}$ and $V \alpha$ agree on the image of $\varphi_{A}$, i.e. the diagram

$$
T V A \stackrel{\varphi_{A}}{\longrightarrow} V \hat{T} A \stackrel{V \underset{V \alpha}{\longrightarrow}}{\stackrel{V \alpha^{\prime}}{\longrightarrow}} V A
$$

commutes. By Proposition B.7(b) the morphism $\varphi_{A}$ is dense, which implies $V \alpha^{\prime}=V \alpha$ since $A$ is a Hausdorff space. Since the forgetful functor $V$ is faithful, we conclude $\alpha=\alpha^{\prime}$.

Proof (Lemma 4.4). Let us first consider the unordered case. Since intersections of pseudovarieties are pseudovarieties, we may assume that our class is presented by a single equation $u=v$ over a finite set $X$.

(a) Closure under finite products: clearly the trivial one-element $\mathbf{T}$-algebra satisfies all profinite equations. Let $\left(A_{0}, \alpha_{0}\right)$ and $\left(A_{1}, \alpha_{1}\right)$ be finite $\mathbf{T}$-algebras satisfying $u=v$ and let $p_{i}:\left(A_{0} \times A_{1}, \alpha\right) \rightarrow\left(A_{i}, \alpha_{i}\right)$ be their product. For any T-homomorphism $h: T \psi_{X} \rightarrow A_{0} \times A_{1}$ put $h_{i}=p_{i} \cdot h$. Then

$$
p_{i} \cdot h^{+}(u)=h_{i}^{+}(u)=h_{i}^{+}(v)=p_{i} \cdot h^{+}(v)
$$

so $h^{+}(u)=h^{+}(v)$ since the projections $p_{i}$ are jointly monic. We conclude that $\left(A_{0} \times A_{1}, \alpha\right)$ satisfies $u=v$. 
(b) Closure under subalgebras: let $(A, \alpha)$ be a finite $\mathbf{T}$-algebra satisfying $u=v$ and $m:(B, \beta) \longmapsto(A, \alpha)$ be a subalgebra. Then for any T-homomorphism $h: T \psi_{X} \rightarrow B$ we have

$$
m \cdot h^{+}(u)=(m \cdot h)^{+}(u)=(m \cdot h)^{+}(v)=m \cdot h^{+}(v)
$$

so $h^{+}(u)=h^{+}(v)$ since $m$ is monic. Hence $(B, \beta)$ satisfies $u=v$.

(c) Closure under quotients: let $(A, \alpha)$ be a finite $\mathbf{T}$-algebra satisfying $u=v$ and $e:(A, \alpha) \rightarrow(B, \beta)$ be a quotient. For any $h_{0}: \psi_{X} \rightarrow B$ choose a morphism $h_{0}^{\prime}: \psi_{X} \rightarrow A$ with $h_{0}=e \cdot h_{0}^{\prime}$, cf. Remark 2.9. The corresponding T-homomorphisms $h: T \psi_{X} \rightarrow B$ and $h^{\prime}: T \psi_{X} \rightarrow A$ satisfy $h=e \cdot h^{\prime}$, so

$$
h^{+}(u)=e \cdot\left(h^{\prime}\right)^{+}(u)=e \cdot\left(h^{\prime}\right)^{+}(v)=h^{+}(v),
$$

proving that $(B, \beta)$ satisfies $u=v$.

The ordered case in analogous: replace profinite equations by inequations, in (a) use that the projections $p_{i}$ are jointly order-reflecting, and in (b) use that $m$ is order-reflecting.

Proof (Lemma 4.11). Consider first the unordered case. For any T-homomorphism $h: T X \rightarrow A$ with $(A, \alpha) \in \mathcal{V}$ we have the commutative triangle (4.1). Since the projections $h_{\mathcal{V}}^{+}$are jointly monic, it follows that $\varphi_{\hat{X}}^{\mathcal{V}} u=\varphi_{\hat{X}}^{\mathcal{V}} v$ iff $h^{+} u=h^{+} v$ for all $h$, i.e. iff every $(A, \alpha) \in \mathcal{V}$ satisfies $u=v$. For the ordered case replace equations by inequations and use that the projections $h_{\mathcal{V}}^{+}$are jointly order-reflecting.

Proof (Theorem 5.5). $3 \Rightarrow 1$ requires a routine verification analogous to the proof of Lemma 4.4, and $1 \Rightarrow 2$ is Theorem 4.12. For $2 \Rightarrow 3$ consider first the unordered case. We may assume that $\mathcal{V}$ is presented by a single profinite equation $u=v$ with $u, v$ elements of some $X \in \mathscr{D}_{f}$. Express $X$ as a quotient $q: \Phi_{Y} \rightarrow X$ for some finite set $Y$. Let $\left\{\left(u_{i}, v_{i}\right): i \in I\right\}$ be the kernel of $\hat{q}: \hat{\Phi_{Y}} \rightarrow X$ (consisting of all pairs $\left(u_{i}, v_{i}\right) \in \hat{\Phi_{Y}} \times \hat{\Phi_{Y}}$ with $\left.\hat{q}\left(u_{i}\right)=\hat{q}\left(v_{i}\right)\right)$, and choose $u^{\prime}, v^{\prime} \in \hat{\Phi_{Y}}$ with $\hat{q}\left(u^{\prime}\right)=u$ and $\hat{q}\left(v^{\prime}\right)=v$. We claim that a finite object $A \in \mathscr{D}_{f}$ satisfies the profinite equation $u=v$ iff it satisfies the profinite implication

$$
\bigwedge_{i \in I} u_{i}=v_{i} \Rightarrow u^{\prime}=v^{\prime}
$$

which proves that $\mathcal{V}$ is presented by that implication.

For the "if" direction suppose that $A$ satisfies (5.3). For any morphism $h: X \rightarrow A$ in $\mathscr{D}$ we have the commutative triangle below:

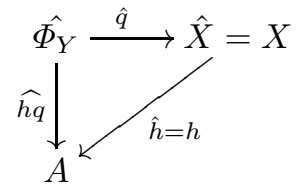


The morphism $\widehat{h q}$ merges $u_{i}, v_{i}$ for all $i$ since $\hat{q}$ does, so by (5.3) it also merges $u^{\prime}, v^{\prime}$. We conclude

$$
\hat{h}(u)=\hat{h} \hat{q}\left(u^{\prime}\right)=\widehat{h q}\left(u^{\prime}\right)=\widehat{h q}\left(v^{\prime}\right)=\hat{h} \hat{q}\left(v^{\prime}\right)=\hat{h}(v),
$$

so $A$ satisfies $u=v$.

For the "only if" direction, suppose $A$ satisfies $u=v$, and let $h: \Phi_{Y} \rightarrow A$ be a morphism with $\hat{h}\left(u_{i}\right)=\hat{h}\left(v_{i}\right)$ for all $i$. Since $\left(u_{i}, v_{i}\right)$ are precisely the pairs merged by $\hat{q}$, the homomorphism theorem (see Remark 2.9) yields a morphism $h^{\prime}: X \rightarrow A$ with $h^{\prime} \cdot \hat{q}=\hat{h}$. Since $A$ satisfies $u=v$, it follows that $h^{\prime}(u)=h^{\prime}(v)$ and hence

$$
\hat{h}\left(u^{\prime}\right)=h^{\prime} \cdot \hat{q}\left(u^{\prime}\right)=h^{\prime}(u)=h^{\prime}(v)=h^{\prime} \cdot \hat{q}\left(v^{\prime}\right)=\hat{h}\left(v^{\prime}\right) .
$$

Hence $A$ satisfies the implication (5.3).

The ordered case is analogous to the above argument, replacing equations by inequations and using the homomorphism theorem for ordered algebras in the "only if" direction. 